\title{
Binary mixtures of 2-ethyl-1-hexanol with various functional groups (benzyl chloride, 3-methylaniline, 3-methoxyaniline and 2,6-dimethylcyclohexanone)
}

\author{
Shaik. Parveen Sulthana ${ }^{1} \cdot$ M. Gowrisankar ${ }^{2} \cdot$ Shaik. Babu $^{3}$ (1) P. Venkateswara Rao ${ }^{4}$
}

Received: 9 March 2020 / Accepted: 15 April 2020 / Published online: 25 April 2020

(c) Springer Nature Switzerland AG 2020

\begin{abstract}
In the binary mixtures of 2-ethyl-1-hexanol with various functional groups (benzyl chloride, 3-methyl aniline, 3-methoxy aniline and 2,6-dimethyl cyclohexanone) densities, speeds of sound and viscosities including those of pure liquids were measured over the entire composition range at different temperatures $(303.15,308.15$ and 313.15$) \mathrm{K}$ and atmospheric pressure $0.1 \mathrm{MPa}$. Using this experimentally determined data, the excess/deviation parameters (molar volume, isentropic compressibility and deviation in viscosity) partial molar volumes, partial molar isentropic compressibilities of these components at infinite dilution were calculated. The results are discussed in terms of intermolecular forces between different component molecules in the binary mixture are more than the average intermolecular forces existing between the similar molecules of pure components.
\end{abstract}

Keywords 2-ethyl-1-hexanol · Binary mixtures · Functional groups

\section{Introduction}

The study of thermodynamic properties of binary mixtures contributes to an understanding of the behavior of different liquids and their functional groups. This information is very useful in the design of industrial process and in the development of theories for the liquid state and predictive methods. Excess thermodynamic parameters of different mixtures are useful in the study of molecular interactions and arrangements.

The knowledge of physicochemical properties of nonaqueous binary liquid mixtures has relevance in theoretical and applied areas of research, and such results are frequently used in design process (flow, mass transfer or heat transfer calculations) in many chemical and industrial processes [1]. The excess properties derived from these physical property data reflect the physicochemical behavior of the liquid mixtures with respect to the solution structure and intermolecular interactions between the component molecules of the mixture $[2,3]$.

Study of hydrogen-bonded systems is essential and helpful as hydrogen bond plays a vital role in chemical, physical and biological process. Organic compounds containing electronegative group can interact with compounds containing active hydrogen through hydrogen bond. This type of hydrogen bond takes part in role in the stability of biologically important molecules. Alkanols are polar and self-associated liquids and the dipolar association of alkanols decreases when they are mixed with polar compounds containing various functional groups due to formation of hydrogen bonds between 2-ethyl1 -hexanol and various functional groups in the mixtures

M. Gowrisankar, mgsankar965@gmail.com | 'Department of Chemistry, Acharya Nagarjuna University, Guntur, AP 522510, India. ${ }^{2}$ Department of Chemistry, J.K.C.C. Acharya Nagarjuna University, Guntur, AP 522006, India. ${ }^{3}$ Department of Physics, Koneru Lakshmaiah Education Foundation, Vaddeswaram, Guntur, AP, India. ${ }^{4}$ Department of Chemistry, RVR \& JC College of Engineering, Chowdavaram, Guntur, AP, India. 
over the rupture of hydrogen bonds present in pure 2-ethyl-1-hexanol.

We report here a study of excess/deviation functions in four non-component molecules with 2-ethyl-1-hexanol systems. This is in continuation of our earlier work [4-6] on excess/deviation functions of polar liquids with amines. However, in this work a systematic study is reported on excess/deviation functions of non-component molecules with 2-ethyl-1-hexanol over the entire composition range and at temperature $(T)$ ranging from 303.15 to $313.15 \mathrm{~K}$. The results are discussed in terms of intermolecular forces between the different components molecules in the binary mixture are more than the average intermolecular forces existing between the similar molecules of pure components.

\section{Procedure}

\subsection{Materials}

The 3-methoxyaniline, benzyl chloride and 3-methylaniline liquids were sigma Aldrich samples. Table 1 contains information regarding their source, purification method, final purity and analysis method. Values of density, speed of sound, and viscosity are presented in Table 2 . These values are in good agreement with the data available in the literature [7-14].

\subsection{Apparatus and procedure}

All the binary liquid mixtures were prepared by weighing required amounts of pure liquids in an electric balance (ER$120 \mathrm{~A}$, Afoset) with a precision of $\pm 0.01 \mathrm{mg}$ by syringing each component into air-tight stopper bottles to minimize evaporation losses. The uncertainty of the mole fraction was $\pm 1 \times 10^{-4}$.

The details of the density, speed of sound and viscosity methods and their measurement techniques were described elsewhere [15]. The uncertainty of density measurement for liquid mixtures is $\pm 0.2 \times 10^{-4} \mathrm{~g} \mathrm{~cm}^{3}$. The uncertainty in the measured speed of sound is $\pm 0.053 \mathrm{~m} \mathrm{~s}^{-1}$. The experimental uncertainty of viscosity estimated as $\pm 1.13 \%$. The temperature of the liquids during the measurements was maintained within an uncertainty of $\pm 0.01 \mathrm{~K}$ in an electronically controlled thermostatic water bath.

\subsection{Theory}

The excess thermodynamic/deviation functions were calculated by using the following equations

$V^{E}=V-\left(x_{1} V_{1}+x_{2} V_{2}\right)$

$\Delta h=h-\left(x_{1} h_{1}+x_{1} h_{1}\right)$

$G^{* E}=R T\left[\ln h V-\left(x_{1} \ln h_{1} V_{1}+x_{2} \ln h_{2} V_{2}\right)\right.$

where $\eta, \eta_{1}, \eta_{2}, V, v_{1}$ and $V_{2}$ are viscosities and mixture molar volumes and pure components respectively.

The isentropic compressibilities were calculated from the relation

$\kappa_{s}=\left(u^{2} \rho\right)^{-1}$

where $\rho$ is the density and $u$ is the speed of sound of the binary mixture.

Further, the excess isentropic compressibilities $\left(k_{s}^{E}\right)$ are calculated from the following relations recommended by Benson and Kiyohara [16]

$k_{s}^{E}=k_{s}-k_{s}^{i d}$

$k_{s}^{i d}=\sum_{i=1}^{2} \varphi_{i}\left[\kappa_{s, i}+\frac{T V_{i}\left(\alpha_{i}^{2}\right)}{C_{p, i}}\right]-\left\{\frac{T\left(\sum_{i=1}^{2} x_{i} V_{i}\right)\left(\sum_{i=1}^{2} \varphi_{i} \alpha_{i}\right)^{2}}{\sum_{i=1}^{2} x_{i} C_{p, i}}\right\}$

Table 1 List of chemicals with details of source, CAS number, purity and water content

\begin{tabular}{|c|c|c|c|c|c|c|}
\hline Name of the chemical & Source & CAS number & Purification method & $\begin{array}{l}\text { Mass frac- } \\
\text { tion purity }\end{array}$ & $\begin{array}{l}\text { Water } \\
\text { content }^{b} \\
(\%)\end{array}$ & $\begin{array}{l}\text { Analysis } \\
\text { method }^{\mathrm{a}}\end{array}$ \\
\hline 2-Ethyl-1-hexanol & Sigma Aldrich, India & $104-76-7$ & None & 0.996 & 0.045 & GC \\
\hline Benzyl chloride & S.D. fine Chemicals, India & $536-90-3$ & Fractional distillation & 0.993 & 0.039 & GC \\
\hline 3-Methylaniline & S.D. fine Chemicals, India & $108-44-1$ & None & 0.996 & 0.042 & GC \\
\hline 3-Methoxyaniline & Sigma Aldrich, India & $536-90-3$ & None & 0.995 & 0.042 & GC \\
\hline 2,6-Dimethylcyclohexanone & S.D. fine Chemicals, India & $2816-57-1$ & None & 0.995 & 0.043 & GC \\
\hline
\end{tabular}

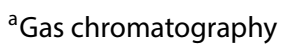

${ }^{\text {b}}$ Karl-Fischer method 
Table 2 Densities, viscosity and speeds of sounds data of pure components at different temperatures and $0.1 \mathrm{MPa}$ pressure

\begin{tabular}{|c|c|c|c|c|c|c|c|}
\hline \multirow[t]{2}{*}{ Component (K) } & \multicolumn{2}{|c|}{ Density $\left(\rho / \mathrm{g} \mathrm{cm}^{-3}\right)$} & \multicolumn{2}{|c|}{ Speed of sound $\left(\mathrm{u} / \mathrm{m} \mathrm{s}^{-1}\right)$} & \multirow[b]{2}{*}{$C_{P}\left(J^{-1} \mathrm{~mol}^{-1}\right)$} & \multicolumn{2}{|c|}{ Viscosity ( $\eta / m P a ~ s)$} \\
\hline & Experimental & Literature & Experimental & Literature & & Experimental & Literature \\
\hline \multicolumn{8}{|l|}{ 2-Ethyl-1-hexanol } \\
\hline \multirow[t]{2}{*}{303.15} & 0.82529 & $0.82539[7]$ & 1302 & $1301[7]$ & $317.5^{\mathrm{a}}[27]$ & 7.146 & 7.144 [7] \\
\hline & & $0.82528[8]$ & & & & & \\
\hline \multirow[t]{2}{*}{308.15} & 0.82159 & $0.82168[7]$ & 1282 & $1283[7]$ & & 6.012 & $6.090[7]$ \\
\hline & & $0.82158[8]$ & & & & & \\
\hline 313.15 & 0.81788 & & 1261 & & & 4.774 & - \\
\hline \multicolumn{8}{|l|}{ Benzyl chloride } \\
\hline 303.15 & 1.09492 & $1.0948[14]$ & 1357.6 & $1356.6[14]$ & $182.4^{\mathrm{a}}[28]$ & 1.114 & $1.1121[14]$ \\
\hline 308.15 & 0.84892 & & 1342.5 & & & 1.058 & \\
\hline 313.15 & 0.60292 & & 1327.4 & & & 1.002 & \\
\hline \multicolumn{8}{|l|}{ 3-Methylaniline } \\
\hline \multirow[t]{2}{*}{303.15} & 0.98032 & $0.9808[10]$ & 1568.2 & $1567.0[10]$ & $228.0[11,12]$ & 3.018 & \\
\hline & & $0.98096[12]$ & & & & & \\
\hline 308.15 & 0.97611 & $0.9727[10]$ & 1530.6 & $1533.0[10]$ & $230 . .0[11,12]$ & 2.707 & \\
\hline 313.15 & 0.97186 & $0.9646[10]$ & 1491.7 & $1492.0[10]$ & $232.0[11,12]$ & 2.396 & \\
\hline \multicolumn{8}{|l|}{ 3-Methoxyaniline } \\
\hline 303.15 & 1.09025 & & 1579.9 & & $228.0[11]$ & 5.593 & \\
\hline 308.15 & 1.08705 & & 1552.0 & & $230.5[11]$ & 5.160 & \\
\hline 313.15 & 1.08320 & & 1523.4 & & $232.6[11]$ & 4.733 & \\
\hline \multicolumn{8}{|c|}{ 2,6-Dimethyl cyclohexanone } \\
\hline 303.15 & $0.90890[13]$ & & $1305.4[13]$ & & $195.2[9]$ & $3.709[13]$ & \\
\hline 308.15 & $0.90460[13]$ & & $1285.1[13]$ & & $197.5[9]$ & $3.237[13]$ & \\
\hline 313.15 & $0.90040[13]$ & & $1265.2[13]$ & & $199.6[9]$ & $2.765[13]$ & \\
\hline
\end{tabular}

The standard uncertainties are $\left(x_{1}\right)=1 \times 10^{-4}, u(\rho) \pm 0.2 \times 10^{-4} \mathrm{~g} \mathrm{~cm}^{-3}, \mathrm{u}(\mathrm{u})=0.5 \%, \mathrm{u}(\eta)=1.03 \%, \mathrm{u}(\mathrm{T})=0.01 \mathrm{~K}$ for density, viscosity and speed of sound, $u(T)=0.02 \mathrm{~K}$ for viscosity and $u(p)=1 \mathrm{kPa}$

a $298.15 \mathrm{~K}$

where $\varphi_{\mathrm{i}}, C_{p, i}, V_{i}, \kappa_{\mathrm{s}, i}$ and $\alpha_{i}$ are the volume fraction, molar heat capacity, molar volume, isentropic compressibility and coefficient of isobaric thermal expansion of pure components respectively.

Excess/deviation functions $\left(V^{E}, \Delta \eta\right.$ and $\left.\kappa_{s}^{E}\right)$ values are fitted to a Redlich-Kister polynomial equation [17]

$Y^{\mathrm{E}}=x_{1} x_{2} \sum_{i=0}^{j} A_{i}\left(1-2 x_{1}\right)^{i}$

where $Y^{E}$ is the $V^{E}, \Delta \eta$ and $\kappa_{s}^{E}$. Coefficientsn values $A_{i}$ are determined by using the method of least-squares. The standard deviation $\sigma\left(Y^{E}\right)$ were calculated by the formula as follows

$\sigma\left(Y^{E}\right)=\left[\Sigma\left(Y_{\text {exp }}^{E}-Y_{c a l}^{E}\right)^{2} /(m-n)\right]^{1 / 2}$ where $m$ is the total number of experimental points and $n$ is the number of parameters. The coefficients, $A_{i}$ and corresponding standard deviation values $(\sigma)$ are presented in Table 3.

\section{Results and discussion}

The experimental density, speed of sound and viscosity data and their excess/deviation values for the 2-ethyl-1-hexanol with various functional groups at various temperatures are given in Tables 2 and 4 . All the excess/deviation values of these parameters $V^{E} K_{s}^{E} \Delta \eta$ and $G^{*} E$ at various temperatures are represented graphically in Figs. 1, 2, 3 and 4. 
Table 3 Coefficients of Redlich-Kister equation and standard deviation $(\sigma)$ values for liquid mixtures of 2-ethyl-1-hexanol with various functional groups at $\mathrm{T}=(303.15-313.15) \mathrm{K}$

\begin{tabular}{|c|c|c|c|c|}
\hline $\mathrm{T}(\mathrm{K})$ & $A_{0}$ & $A_{1}$ & $A_{2}$ & $\sigma$ \\
\hline \multicolumn{5}{|c|}{ 2-Ethyl-1-hexanol (1) + benzyl chloride (2) } \\
\hline \multicolumn{5}{|c|}{$V^{E}\left(\mathrm{~cm}^{3} \mathrm{~mol}^{-1}\right)$} \\
\hline 303.15 & -0.4085 & -0.4731 & -0.5329 & 0.001 \\
\hline 308.15 & 0.0642 & 0.0887 & 0.1081 & 0.001 \\
\hline 313.15 & 0.0317 & -0.1143 & -0.2181 & 0.001 \\
\hline \multicolumn{5}{|c|}{$\kappa_{s}^{E}\left(\mathrm{TPa}^{-1}\right)$} \\
\hline 303.15 & -77.93 & -0.638 & 21.61 & 0.001 \\
\hline 308.15 & -79.94 & 0.559 & 7.987 & 0.066 \\
\hline 313.15 & -81.94 & 1.755 & -5.625 & 0.002 \\
\hline \multicolumn{5}{|c|}{$\Delta \eta(\mathrm{mPa} s)$} \\
\hline 303.15 & 0.101 & -0.019 & -0.005 & 0.001 \\
\hline 308.15 & 0.105 & -0.026 & 0.011 & 0.001 \\
\hline 313.15 & 0.107 & -0.019 & 0.031 & 0.001 \\
\hline \multicolumn{5}{|c|}{ 2-Ethyl-1-hexanol (1) + 3-methylaniline (2) } \\
\hline \multicolumn{5}{|c|}{$V^{E}\left(\mathrm{~cm}^{3} \mathrm{~mol}^{-1}\right)$} \\
\hline 303.15 & -0.4201 & -0.4853 & -0.5468 & 0.001 \\
\hline 308.15 & 0.0636 & 0.0877 & 0.1017 & 0.001 \\
\hline 313.15 & -0.0021 & -0.1404 & -0.2746 & 0.001 \\
\hline \multicolumn{5}{|c|}{$\kappa_{s}^{E}\left(\mathrm{TPa}^{-1}\right)$} \\
\hline 303.15 & -80.69 & 16.24 & -14.09 & 0.001 \\
\hline 308.15 & -82.69 & 17.78 & -23.47 & 0.134 \\
\hline 313.15 & -84.25 & 16.48 & -38.10 & 0.104 \\
\hline \multicolumn{5}{|c|}{$\Delta \eta(\mathrm{mPa} s)$} \\
\hline 303.15 & 0.051 & -0.031 & 0.007 & 0.001 \\
\hline 308.15 & 0.053 & -0.034 & 0.014 & 0.001 \\
\hline 313.15 & 0.0547 & -0.038 & 0.024 & 0.001 \\
\hline \multicolumn{5}{|c|}{ 2-Ethyl-1-hexanol (1) + 3-methoxyaniline (2) } \\
\hline \multicolumn{5}{|c|}{$V^{E}\left(\mathrm{~cm}^{3} \mathrm{~mol}^{-1}\right)$} \\
\hline 303.15 & -0.4262 & -0.4954 & -0.5649 & 0.001 \\
\hline 308.15 & 0.0767 & 0.0904 & 0.1037 & 0.001 \\
\hline 313.15 & -0.0705 & -0.1964 & -0.3210 & 0.001 \\
\hline \multicolumn{5}{|c|}{$\kappa_{s}^{E}\left(\mathrm{TPa}^{-1}\right)$} \\
\hline 303.15 & -82.98 & 16.71 & -20.96 & 0.001 \\
\hline 308.15 & -84.37 & 17.37 & -31.84 & 0.001 \\
\hline 313.15 & -86.56 & 16.47 & -43.24 & 0.109 \\
\hline \multicolumn{5}{|c|}{$\Delta \eta(\mathrm{mPa} s)$} \\
\hline 303.15 & 0.107 & -0.018 & 0.231 & 0.001 \\
\hline 308.15 & 0.111 & -0.025 & 0.031 & 0.001 \\
\hline 313.15 & 0.115 & -0.018 & 0.049 & 0.001 \\
\hline \multicolumn{5}{|c|}{ 2-Ethyl-1-hexanol (1) +2,6-dimethylcyclohexanone (2) } \\
\hline \multicolumn{5}{|c|}{$V^{E}\left(\mathrm{~cm}^{3} \mathrm{~mol}^{-1}\right)$} \\
\hline 303.15 & -0.4353 & -0.5167 & -0.5861 & 0.001 \\
\hline 308.15 & 0.0812 & 0.0848 & 0.1068 & 0.001 \\
\hline 313.15 & -0.1282 & -0.2206 & -0.3707 & 0.001 \\
\hline \multicolumn{5}{|c|}{$\kappa_{s}^{E}\left(\mathrm{TPa}^{-1}\right)$} \\
\hline 303.15 & -14.061 & -2.154 & 0.898 & 0.005 \\
\hline 308.15 & -14.339 & -2.246 & -0.270 & 0.001 \\
\hline 313.15 & -14.617 & -2.340 & -1.445 & 0.005 \\
\hline
\end{tabular}

Table 3 (continued)

\begin{tabular}{lllll}
\hline $\mathrm{T}(\mathrm{K})$ & $\mathrm{A}_{0}$ & $\mathrm{~A}_{1}$ & $\mathrm{~A}_{2}$ & $\sigma$ \\
\hline$\Delta \eta$ (mPa s) & & & & \\
303.15 & 0.112 & -0.017 & 0.036 & 0.001 \\
308.15 & 0.114 & -0.025 & 0.051 & 0.001 \\
313.15 & 0.119 & -0.016 & 0.070 & 0.001 \\
\hline
\end{tabular}

All the excess/deviation values of these parameters may be explained qualitatively in terms of

1. molecular interactions between like/unlike molecules and the difference in size and shape of the unlike components

2. Possible $\mathrm{H}$-bond interaction between 2-ethyl-1-hexanol and various functional groups in the mixtures having 2-ethyl-1-hexanol as a proton donor

3. Dipole-dipole interactions between polar-polar components

The magnitude of excess values of $V^{E}$ and $\kappa_{s}^{E}$ from ideality of the systems that can be negative, positive, or zero may be explained as a balance between positive contributions (hydrogen bond rupture and dispersive interactions between unlike molecules) and negative contributions (intermolecular dipolar interactions, geometrical fitting between components, intermolecular association complexes between unlike molecules)

The experimental values indicate that the negative effects are dominant over positive ones in all the mixtures, since the excess values of $V^{E}$ and $\kappa_{s}^{E}$ for all the binary systems is negative over the whole concentration range at various temperatures. In the present systems, negative sign of $\mathrm{V}^{\mathrm{E}}$ shows that the predominance of formation of hydrogen bonds between 2-ethyl-1-hexanol and various functional groups in the mixtures over the rupture of hydrogen bonds present in pure 2-ethyl1-hexanol [18, 19].

The negative values of $\mathrm{V}^{\mathrm{E}}$ for the four systems are in the following order:

2-Ethyl-1-hexanol +2, 6-dimethylcyclohexanone $>2$-ethyl-1-hexanol + 3-methoxyaniline $>2$-ethyl-1-hexanol +3-methylaniline $>2$-ethyl1-hexanol + benzyl chloride

The value at the minima for the system (2-ethyl-1-hexanol +2,6-dimethylcyclohexanone) is slightly more negative than that of remaining various functional groups, and this may be due to the ability of 2,6-dimethylcyclohexanone to form stronger hydrogen bonds with 2-ethyl-1-hexanol than various functional groups with 
Table 4 Density $(\rho)$, excess molar volumes $\left(V^{E}\right)$, speed of sound $(u)$, excess isentropic compressibility $\left(\kappa_{s}^{E}\right)$, viscosity $(\eta)$, deviation in viscosity $(\Delta \eta)$ and excess Gibbs energy of activation of viscous flow
$\left(G^{*} E\right)$ of binary liquid mixtures of 2-ethyl-1-hexanol with various functional groups at $\mathrm{T}=(303.15$ to 313.15$) \mathrm{K}$ and $0.1 \mathrm{MPa}$ pressure

\begin{tabular}{llllll}
\hline $\mathrm{x}_{1}$ & Density $(\rho)\left(\mathrm{g} \mathrm{cm}^{-3}\right)$ & $V^{E}\left(\mathrm{~cm}^{3} \mathrm{~mol}^{-1}\right)$ & $u\left(\mathrm{~m} \mathrm{~s}^{-1}\right)$ & $\kappa_{s}^{E}\left(\mathrm{TPa}^{-1}\right)$ & $\begin{array}{l}\text { Viscosity }(\eta) \\
(\mathrm{mPa} \mathrm{s})\end{array}$ \\
\hline
\end{tabular}

2-Ethyl-1-hexanol (1)++benzyl chloride (2)

$303.15 \mathrm{~K}$

\subsection{0}

0.0000

0.0905

0.1896

0.2577

0.3423

0.4351

0.5296

0.6229

0.7201

0.8312

1.0000

$308.15 \mathrm{~K}$

$0.0000 \quad 0.84892$

$0.0905 \quad 0.84659$

$0.1896 \quad 0.84396$

0.2577

0.3423

0.4351

0.5296

0.6229

0.7201

0.8312

0.84213

0.83982

0.83727

0.83466

0.83208

0.82940

1.0000

0.82631

$313.15 \mathrm{~K}$

$0.0000 \quad 0.60292$

$0.0905 \quad 0.61819$

0.1896

0.2577

0.3423

0.4351

0.5296

0.6229

0.7201

0.8312

1.0000

0.63565

$$
0.64812
$$

$$
0.66421
$$

$$
0.68268
$$

0.70243

0.72296

0.74550

0.77284

0.81788

2-Ethyl-1-hexanol (1) + 3-methylaniline (2)

$303.15 \mathrm{~K}$

\begin{tabular}{ll}
0.0000 & 0.98032 \\
0.0951 & 0.96024 \\
0.1616 & 0.94708 \\
0.2417 & 0.93211 \\
0.3403 & 0.91486 \\
0.4388 & 0.89884 \\
0.5389 & 0.88365 \\
0.6281 & 0.87097 \\
\hline
\end{tabular}

$$
\begin{array}{r}
0.0000 \\
-0.0362 \\
-0.0670 \\
-0.0827 \\
-0.0958 \\
-0.1023 \\
-0.1008 \\
-0.0918 \\
-0.0754 \\
-0.0494 \\
0.0000
\end{array}
$$

$$
0.0000
$$$$
-0.0512
$$$$
-0.0872
$$$$
-0.1039
$$$$
-0.1145
$$$$
-0.1195
$$$$
-0.1165
$$$$
-0.1066
$$$$
-0.0921
$$$$
-0.0648
$$$$
0.0000
$$$$
0.0000
$$$$
-0.0633
$$$$
-0.1051
$$$$
-0.1214
$$$$
-0.1325
$$$$
-0.1353
$$$$
-0.1315
$$$$
-0.1221
$$$$
-0.1067
$$$$
\begin{array}{r}
-0.0779 \\
0.0000
\end{array}
$$$$
\begin{array}{r}
-0.0779 \\
0.0000
\end{array}
$$

$$
\text { (2) }
$$

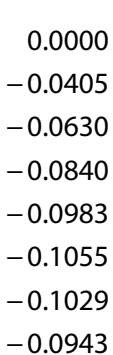

$$
\begin{aligned}
& 1357.0 \\
& 1349.0 \\
& 1342.8 \\
& 1339.5 \\
& 1335.7 \\
& 1331.5 \\
& 1327.0 \\
& 1322.2 \\
& 1316.7 \\
& 1310.4 \\
& 1302.0
\end{aligned}
$$

\section{0}

1342.0

1341.7

1340.5

1338.9

1336.1

1331.7

1326.1

1319.3

1310.8

1300.0

1282.0

1327.2

1324.3

1320.3

1317.0

1312.5

1306.8

1300.5

1293.8

1286.2

1276.8

1261.0

1568.2
1529.0
1504.0
1476.2
1445.3
1417.8
1392.8
1372.6

0.000
-5.179
-10.63
-13.88
-17.02
-19.05
-19.41
-18.04
-14.92
-9.664
0.000

\subsection{4}

1.669

2.275

2.689

3.203

3.764

4.333

4.894

5.476

6.140

7.146

$0.000 \quad 1.058$

$-6.180$

$-11.87$

$-14.96$

$-17.91$

$-19.51$

$-19.93$

$-18.71$

$-15.74$

$-10.66$

0.000

1.516

2.016

2.357

2.779

3.240

3.708

4.168

4.645

5.189

6.012

0.000

$-7.174$

1.002

1.355

$-13.09$

1.737

$-16.09$

$-18.70$

1.997

2.319

$-20.22$

$-20.39$

$-19.23$

$-16.58$

$-11.68$

0.000

2.670

3.027

3.377

3.739

4.152

4.774

0.000
-8.867
-13.29
-17.01
-19.60
-20.41
-19.76
-18.09

\begin{tabular}{l}
0.000 \\
0.009 \\
0.017 \\
0.020 \\
0.024 \\
0.025 \\
0.025 \\
0.023 \\
0.019 \\
0.012 \\
0.000 \\
\\
0.000 \\
0.010 \\
0.018 \\
0.022 \\
0.025 \\
0.026 \\
0.026 \\
0.024 \\
0.020 \\
0.013 \\
0.000 \\
\hline \\
0.000 \\
0.012 \\
0.020 \\
0.023 \\
0.026 \\
0.027 \\
0.027 \\
0.025 \\
0.021 \\
0.015 \\
0.000
\end{tabular}

0.000

5.981

9.180

10.24

10.71

10.46

9.590

8.282

6.543

4.181

0.000

0.000

5.124

7.965

8.925

9.375

9.180

8.440

7.301

5.773

3.691

0.000

0.000

4.192

6.662

7.555

8.034

7.957

7.393

6.451

5.144

3.320

0.000

$\begin{array}{lll}3.018 & 0.000 & 0.000 \\ 3.421 & 0.011 & 1.233 \\ 3.701 & 0.016 & 1.846 \\ 4.037 & 0.021 & 2.363 \\ 4.448 & 0.025 & 2.721 \\ 4.856 & 0.026 & 2.819 \\ 5.268 & 0.026 & 2.697 \\ 5.635 & 0.024 & 2.425\end{array}$


Table 4 (continued)

\begin{tabular}{|c|c|c|c|c|c|c|c|}
\hline $\mathrm{x}_{1}$ & Density $(\rho)\left(\mathrm{g} \mathrm{cm}^{-3}\right)$ & $V^{E}\left(\mathrm{~cm}^{3} \mathrm{~mol}^{-1}\right)$ & $u\left(\mathrm{~m} \mathrm{~s}^{-1}\right)$ & $\kappa_{s}^{E}\left(\mathrm{TPa}^{-1}\right)$ & $\begin{array}{l}\text { Viscosity (n) } \\
\text { (mPa s) }\end{array}$ & $\Delta \eta(\mathrm{mPa} s)$ & $\mathrm{G}^{* \mathrm{E}}\left(\mathrm{J} \mathrm{mol}^{-1}\right)$ \\
\hline 0.7341 & 0.85684 & -0.0763 & 1350.7 & -14.87 & 6.068 & 0.019 & 1.929 \\
\hline 0.8347 & 0.84429 & -0.0523 & 1331.5 & -10.501 & 6.477 & 0.013 & 1.302 \\
\hline 1.0000 & 0.82529 & 0.0000 & 1302.0 & 0.000 & 7.146 & 0.000 & 0.000 \\
\hline \multicolumn{8}{|c|}{$308.15 \mathrm{~K}$} \\
\hline 0.0000 & 0.97611 & 0.0000 & 1530.6 & 0.000 & 2.707 & 0.000 & 0.000 \\
\hline 0.0951 & 0.95621 & -0.0555 & 1495.6 & -9.728 & 3.033 & 0.012 & 1.110 \\
\hline 0.1616 & 0.94313 & -0.0827 & 1472.5 & -14.30 & 3.258 & 0.017 & 1.660 \\
\hline 0.2417 & 0.92821 & -0.1050 & 1446.3 & -17.87 & 3.528 & 0.022 & 2.134 \\
\hline 0.3403 & 0.91100 & -0.1189 & 1417.2 & -20.22 & 3.858 & 0.026 & 2.466 \\
\hline 0.4388 & 0.89500 & -0.1220 & 1391.3 & -20.91 & 4.184 & 0.027 & 2.560 \\
\hline 0.5389 & 0.87985 & -0.1192 & 1368.0 & -20.43 & 4.515 & 0.027 & 2.460 \\
\hline 0.6281 & 0.86720 & -0.1095 & 1349.0 & -18.74 & 4.808 & 0.025 & 2.218 \\
\hline 0.7341 & 0.85312 & -0.0927 & 1328.5 & -15.56 & 5.154 & 0.021 & 1.768 \\
\hline 0.8347 & 0.84060 & -0.0678 & 1310.3 & -11.14 & 5.480 & 0.014 & 1.199 \\
\hline 1.0000 & 0.82159 & 0.0000 & 1282.0 & 0.000 & 6.012 & 0.000 & 0.000 \\
\hline \multicolumn{8}{|l|}{$313.15 \mathrm{~K}$} \\
\hline 0.0000 & 0.97186 & 0.0000 & 1491.7 & 0.000 & 2.396 & 0.000 & 0.000 \\
\hline 0.0951 & 0.95215 & -0.0697 & 1460.7 & -10.59 & 2.635 & 0.013 & 0.913 \\
\hline 0.1616 & 0.93913 & -0.1001 & 1439.5 & -15.31 & 2.799 & 0.019 & 1.367 \\
\hline 0.2417 & 0.92426 & -0.1238 & 1415.1 & -18.73 & 2.994 & 0.023 & 1.758 \\
\hline 0.3403 & 0.90710 & -0.1366 & 1387.8 & -20.84 & 3.232 & 0.027 & 2.041 \\
\hline 0.4388 & 0.89113 & -0.1385 & 1363.5 & -21.41 & 3.467 & 0.028 & 2.130 \\
\hline 0.5389 & 0.87603 & -0.1354 & 1341.7 & -20.79 & 3.705 & 0.028 & 2.057 \\
\hline 0.6281 & 0.86342 & -0.1247 & 1324.3 & -19.39 & 3.916 & 0.026 & 1.864 \\
\hline 0.7341 & 0.84939 & -0.1091 & 1305.4 & -16.57 & 4.163 & 0.022 & 1.494 \\
\hline 0.8347 & 0.83691 & -0.0833 & 1288.6 & -12.41 & 4.397 & 0.016 & 1.019 \\
\hline 1.0000 & 0.81788 & 0.0000 & 1261.0 & 0.000 & 4.774 & 0.000 & 0.000 \\
\hline \multicolumn{8}{|c|}{ 2-Ethyl-1-hexanol (1) + 3-methoxyaniline (2) } \\
\hline \multicolumn{8}{|c|}{$303.15 \mathrm{~K}$} \\
\hline 0.0000 & 1.09025 & 0.0000 & 1579.9 & 0.000 & 5.593 & 0.000 & 0.000 \\
\hline 0.0981 & 1.05573 & -0.0472 & 1532.1 & -9.728 & 5.752 & 0.007 & 0.225 \\
\hline 0.1789 & 1.02906 & -0.0741 & 1497.3 & -15.04 & 5.882 & 0.011 & 0.366 \\
\hline 0.2587 & 1.00416 & -0.0920 & 1466.8 & -18.40 & 6.008 & 0.013 & 0.465 \\
\hline 0.3569 & 0.97533 & -0.1042 & 1434.0 & -20.54 & 6.161 & 0.014 & 0.540 \\
\hline 0.4412 & 0.95206 & -0.1075 & 1409.6 & -21.01 & 6.292 & 0.013 & 0.564 \\
\hline 0.5309 & 0.92866 & -0.1050 & 1387.0 & -20.43 & 6.429 & 0.012 & 0.554 \\
\hline 0.6399 & 0.90198 & -0.0945 & 1363.2 & -18.42 & 6.596 & 0.010 & 0.497 \\
\hline 0.7458 & 0.87772 & -0.0769 & 1343.2 & -15.13 & 6.758 & 0.007 & 0.398 \\
\hline 0.8498 & 0.85534 & -0.0520 & 1325.6 & -10.408 & 6.917 & 0.004 & 0.260 \\
\hline 1.0000 & 0.82529 & 0.0000 & 1302.0 & 0.000 & 7.146 & 0.000 & 0.000 \\
\hline \multicolumn{8}{|c|}{$308.15 \mathrm{~K}$} \\
\hline 0.0000 & 1.08705 & 0.0000 & 1552.0 & 0.000 & 5.160 & 0.000 & 0.000 \\
\hline 0.0981 & 1.05254 & -0.0615 & 1506.2 & -10.52 & 5.251 & 0.008 & 0.190 \\
\hline 0.1789 & 1.02582 & -0.0932 & 1472.3 & -15.96 & 5.324 & 0.012 & 0.307 \\
\hline 0.2587 & 1.00086 & -0.1121 & 1442.2 & -19.21 & 5.394 & 0.014 & 0.389 \\
\hline 0.3569 & 0.97195 & -0.1233 & 1409.9 & -21.10 & 5.479 & 0.015 & 0.449 \\
\hline 0.4412 & 0.94861 & -0.1254 & 1386.0 & -21.41 & 5.550 & 0.014 & 0.468 \\
\hline 0.5309 & 0.92517 & -0.1222 & 1364.0 & -20.78 & 5.625 & 0.013 & 0.459 \\
\hline 0.6399 & 0.89844 & -0.1119 & 1341.2 & -18.90 & 5.715 & 0.010 & 0.409 \\
\hline
\end{tabular}


Table 4 (continued)

\begin{tabular}{|c|c|c|c|c|c|c|c|}
\hline $\mathrm{x}_{1}$ & Density $(\rho)\left(\mathrm{g} \mathrm{cm}^{-3}\right)$ & $V^{E}\left(\mathrm{~cm}^{3} \mathrm{~mol}^{-1}\right)$ & $u\left(\mathrm{~m} \mathrm{~s}^{-1}\right)$ & $\kappa_{s}^{E}\left(\mathrm{TPa}^{-1}\right)$ & $\begin{array}{l}\text { Viscosity (n) } \\
\text { (mPa s) }\end{array}$ & $\Delta \eta(\mathrm{mPa} s)$ & $\mathrm{G}^{* \mathrm{E}}\left(\mathrm{J} \mathrm{mol}^{-1}\right)$ \\
\hline 0.7458 & 0.87415 & -0.0945 & 1322.1 & -15.84 & 5.803 & 0.008 & 0.327 \\
\hline 0.8498 & 0.85174 & -0.0674 & 1305.2 & -11.21 & 5.889 & 0.005 & 0.213 \\
\hline 1.0000 & 0.82159 & 0.0000 & 1282.0 & 0.000 & 6.012 & 0.000 & 0.000 \\
\hline \multicolumn{8}{|c|}{$313.15 \mathrm{~K}$} \\
\hline 0.0000 & 1.08320 & 0.0000 & 1523.4 & 0.000 & 4.733 & 0.000 & 0.000 \\
\hline 0.0981 & 1.04880 & -0.0757 & 1479.6 & -11.31 & 4.746 & 0.009 & 0.174 \\
\hline 0.1789 & 1.02211 & -0.1122 & 1446.4 & -16.89 & 4.753 & 0.013 & 0.277 \\
\hline 0.2587 & 0.99715 & -0.1323 & 1416.8 & -20.02 & 4.759 & 0.015 & 0.348 \\
\hline 0.3569 & 0.96823 & -0.1425 & 1385.0 & -21.67 & 4.763 & 0.015 & 0.399 \\
\hline 0.4412 & 0.94489 & -0.1433 & 1361.8 & -22.02 & 4.766 & 0.015 & 0.415 \\
\hline 0.5309 & 0.92145 & -0.1394 & 1340.7 & -21.54 & 4.768 & 0.013 & 0.404 \\
\hline 0.6399 & 0.89474 & -0.1293 & 1318.4 & -19.58 & 4.770 & 0.011 & 0.360 \\
\hline 0.7458 & 0.87048 & -0.1121 & 1300.2 & -16.75 & 4.772 & 0.008 & 0.285 \\
\hline 0.8498 & 0.84807 & -0.0829 & 1284.2 & -12.32 & 4.773 & 0.005 & 0.185 \\
\hline 1.0000 & 0.81788 & 0.0000 & 1261.0 & 0.000 & 4.774 & 0.000 & 0.000 \\
\hline \multicolumn{8}{|c|}{ 2-Ethyl-1-hexanol (1) +2,6-dimethylcyclohexanone (2) } \\
\hline \multicolumn{8}{|c|}{$303.15 \mathrm{~K}$} \\
\hline 0.0000 & 0.90890 & 0.0000 & 1305.4 & 0.000 & 3.709 & 0.000 & 0.000 \\
\hline 0.1012 & 0.89975 & -0.0529 & 1305.2 & -1.072 & 4.070 & 0.014 & 0.672 \\
\hline 0.1895 & 0.89186 & -0.0822 & 1305.1 & -1.903 & 4.381 & 0.021 & 1.069 \\
\hline 0.2695 & 0.88481 & -0.0984 & 1305.1 & -2.528 & 4.660 & 0.025 & 1.307 \\
\hline 0.3621 & 0.87676 & -0.1080 & 1305.1 & -3.091 & 4.981 & 0.027 & 1.457 \\
\hline 0.4569 & 0.86868 & -0.1100 & 1305.0 & -3.439 & 5.308 & 0.028 & 1.494 \\
\hline 0.5429 & 0.86149 & -0.1065 & 1304.8 & -3.540 & 5.603 & 0.028 & 1.438 \\
\hline 0.6235 & 0.85486 & -0.0993 & 1304.5 & -3.415 & 5.878 & 0.026 & 1.314 \\
\hline 0.7523 & 0.84451 & -0.0796 & 1303.9 & -2.784 & 6.316 & 0.021 & 0.993 \\
\hline 0.8523 & 0.83665 & -0.0556 & 1303.2 & -1.902 & 6.653 & 0.015 & 0.648 \\
\hline 1.0000 & 0.82529 & 0.0000 & 1302.0 & 0.000 & 7.146 & 0.000 & 0.000 \\
\hline \multicolumn{8}{|c|}{$308.15 \mathrm{~K}$} \\
\hline 0.0000 & 0.90460 & 0.0000 & 1285.1 & 0.000 & 3.237 & 0.000 & 0.000 \\
\hline 0.1012 & 0.89560 & -0.0659 & 1284.9 & -1.157 & 3.533 & 0.015 & 0.635 \\
\hline 0.1895 & 0.88780 & -0.1005 & 1284.8 & -2.004 & 3.785 & 0.023 & 1.006 \\
\hline 0.2695 & 0.88080 & -0.1187 & 1284.8 & -2.630 & 4.011 & 0.026 & 1.224 \\
\hline 0.3621 & 0.87282 & -0.1286 & 1284.8 & -3.174 & 4.270 & 0.028 & 1.362 \\
\hline 0.4569 & 0.86479 & -0.1304 & 1284.7 & -3.511 & 4.534 & 0.029 & 1.398 \\
\hline 0.5429 & 0.85765 & -0.1268 & 1284.5 & -3.607 & 4.772 & 0.029 & 1.346 \\
\hline 0.6235 & 0.85107 & -0.1195 & 1284.3 & -3.500 & 4.994 & 0.027 & 1.232 \\
\hline 0.7523 & 0.84078 & -0.0988 & 1283.8 & -2.896 & 5.347 & 0.023 & 0.937 \\
\hline 0.8523 & 0.83296 & -0.0713 & 1283.2 & -2.021 & 5.618 & 0.016 & 0.614 \\
\hline 1.0000 & 0.82159 & 0.0000 & 1282.0 & 0.000 & 6.012 & 0.000 & 0.000 \\
\hline \multicolumn{8}{|c|}{$313.15 \mathrm{~K}$} \\
\hline 0.0000 & 0.90040 & 0.0000 & 1265.2 & 0.000 & 2.765 & 0.000 & 0.000 \\
\hline 0.1012 & 0.89156 & -0.0834 & 1264.8 & -1.242 & 2.985 & 0.016 & 0.549 \\
\hline 0.1895 & 0.88383 & -0.1211 & 1264.6 & -2.105 & 3.169 & 0.024 & 0.859 \\
\hline 0.2695 & 0.87687 & -0.1389 & 1264.5 & -2.733 & 3.334 & 0.027 & 1.043 \\
\hline 0.3621 & 0.86893 & -0.1486 & 1264.3 & -3.256 & 3.522 & 0.029 & 1.159 \\
\hline 0.4569 & 0.86094 & -0.1494 & 1264.1 & -3.582 & 3.713 & 0.031 & 1.193 \\
\hline 0.5429 & 0.85383 & -0.1449 & 1263.8 & -3.674 & 3.885 & 0.030 & 1.151 \\
\hline 0.6235 & 0.84728 & -0.1368 & 1263.5 & -3.585 & 4.046 & 0.028 & 1.059 \\
\hline
\end{tabular}


Table 4 (continued)

\begin{tabular}{|c|c|c|c|c|c|c|c|}
\hline$x_{1}$ & Density $(\rho)\left(\mathrm{g} \mathrm{cm}^{-3}\right)$ & $V^{E}\left(\mathrm{~cm}^{3} \mathrm{~mol}^{-1}\right)$ & $u\left(\mathrm{~m} \mathrm{~s}^{-1}\right)$ & $\kappa_{s}^{E}\left(\mathrm{TPa}^{-1}\right)$ & $\begin{array}{l}\text { Viscosity (n) } \\
\text { (mPa s) }\end{array}$ & $\Delta \eta(\mathrm{mPa} s)$ & $\mathrm{G}^{* \mathrm{E}}\left(\mathrm{J} \mathrm{mol}^{-1}\right)$ \\
\hline 0.7523 & 0.83706 & -0.1173 & 1262.9 & -3.008 & 4.300 & 0.024 & 0.809 \\
\hline 0.8523 & 0.82926 & -0.0870 & 1262.3 & -2.141 & 4.495 & 0.018 & 0.539 \\
\hline 1.0000 & 0.81788 & 0.0000 & 1261.0 & 0.000 & 4.774 & 0.000 & 0.000 \\
\hline
\end{tabular}

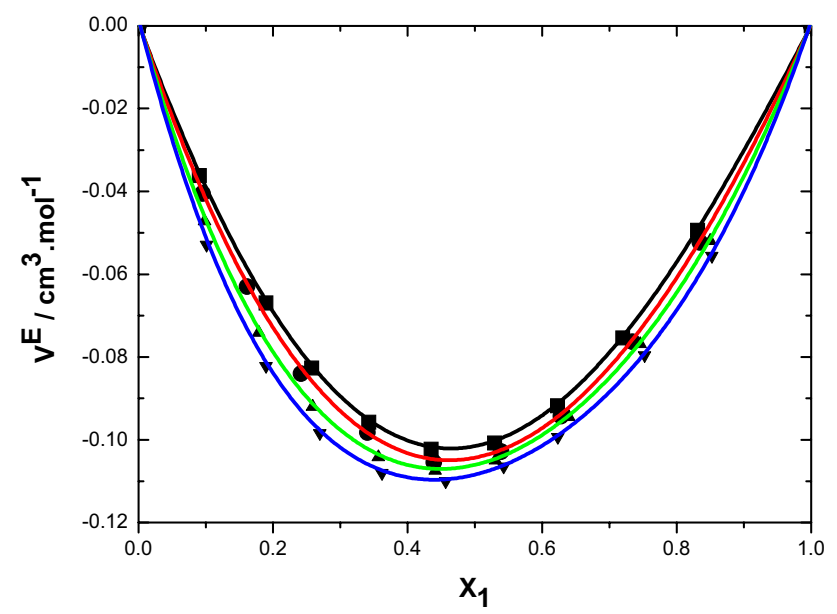

Fig. 1 Curves of excess molar volume $\left(V^{E}\right)$ with mole fraction for the binary mixtures of 2-ethyl-1-hexanol with benzyl chloride (square); 3-methylaniline (circle):3-methoxyaniline (triangle) and 2,6-dimethylcyclohexanone (inverted triangle) at $303.15 \mathrm{~K}$

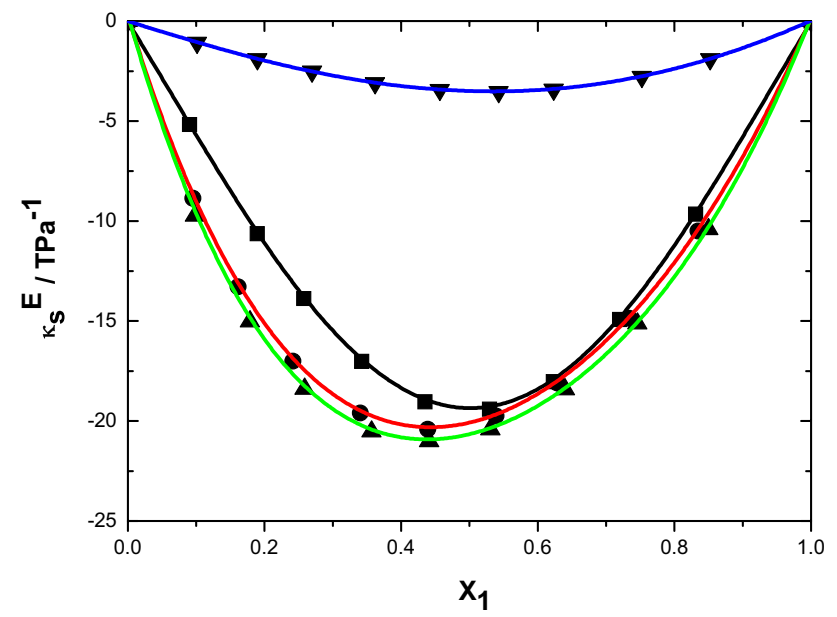

Fig. 2 Curves of excess isentropic compressibility with mole fraction for the binary mixtures of 2-ethyl-1-hexanol with benzyl chloride (square); 3-methylaniline (circle):3-methoxyaniline (triangle) and 2,6-dimethylcyclohexanone (inverted triangle) at $303.15 \mathrm{~K}$

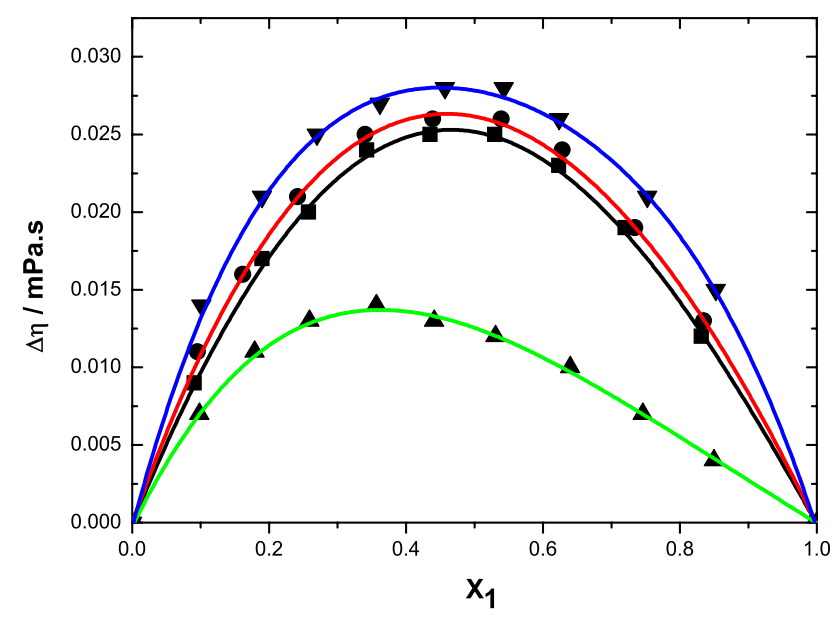

Fig. 3 Variation of deviation in viscosity $(\Delta \eta)$ with mole fraction for the binary mixtures of 2-ethyl-1-hexanol with benzyl chloride (square); 3-methylaniline (circle):3-methoxyaniline (triangle) and 2,6-dimethylcyclohexanone (inverted triangle) at $303.15 \mathrm{~K}$

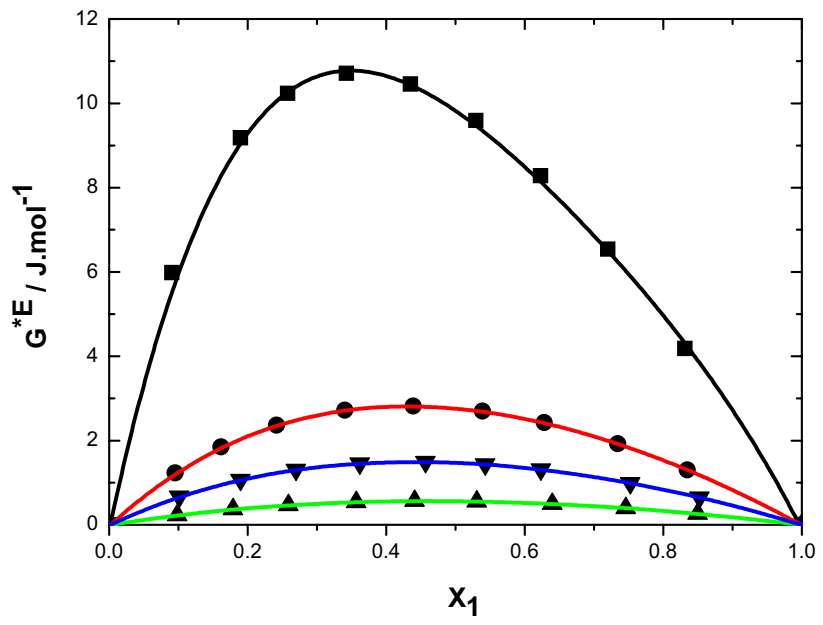

Fig. 4 Excess Gibbs energy of activation of viscous flow $\left(G^{*}\right)$ with mole fraction $\left(x_{1}\right)$ of 2-ethyl-1-hexanol with benzyl chloride (square); 3-methylaniline (circle):3-methoxyaniline (triangle) and 2,6-dimethylcyclohexanone (inverted triangle) at $303.15 \mathrm{~K}$ 
Table 5 The values of $\bar{V}_{\mathrm{m}, 1}^{\circ}$ $V_{\mathrm{m}, 1^{\prime}}^{*}, \bar{V}_{\mathrm{m}, 1^{\prime}}^{\circ \mathrm{E}} \bar{V}_{\mathrm{m}, 2^{\prime}}^{\circ} V_{\mathrm{m}, 2}^{*}$ and $\bar{V}_{\mathrm{m}, 2}{ }^{\circ}$ of the components for 2-ethyl-1-hexanol with various functional groups of binary mixtures at $\mathrm{T}=(303.15-$ 313.15) K

\begin{tabular}{|c|c|c|c|c|c|c|}
\hline $\begin{array}{l}\mathrm{T}(\mathrm{K}) \\
\left(\mathrm{cm}^{3} \mathrm{mc}\right.\end{array}$ & $\bar{V}_{\mathrm{m}, 1}^{0}$ & $V_{\mathrm{m}, 1}^{*}$ & $\bar{V}_{\mathrm{m}, 1}^{\circ} \mathrm{E}$ & $\bar{V}_{\mathrm{m}, 2}^{\circ}$ & $V_{m, 2}^{*}$ & $\bar{V}_{\mathrm{m}, 2}^{\circ} \mathrm{E}$ \\
\hline \multicolumn{7}{|c|}{ 2-Ethyl-1-hexanol(1) + benzyl chloride (2) } \\
\hline 303.15 & 157.49 & 157.80 & -0.313 & 115.17 & 115.61 & -0.441 \\
\hline 308.15 & 158.01 & 158.51 & -0.499 & 148.43 & 149.11 & -0.676 \\
\hline 313.15 & 158.59 & 159.23 & -0.643 & 209.09 & 209.94 & -0.859 \\
\hline \multicolumn{7}{|c|}{ 2-ethyl-1-hexanol(1) + 3-methylaniline (2) } \\
\hline 303.15 & 157.44 & 157.80 & -0.359 & 108.82 & 109.30 & -0.486 \\
\hline 308.15 & 157.97 & 158.51 & -0.538 & 109.06 & 109.77 & -0.713 \\
\hline 313.15 & 158.51 & 159.23 & -0.720 & 109.33 & 110.25 & -0.923 \\
\hline \multicolumn{7}{|c|}{ 2-Ethyl-1-hexanol (1) + 3-methoxy aniline (2) } \\
\hline 303.15 & 157.38 & 157.80 & -0.420 & 112.39 & 112.96 & -0.573 \\
\hline 308.15 & 157.91 & 158.51 & -0.601 & 112.52 & 113.30 & -0.782 \\
\hline 313.15 & 158.45 & 159.23 & -0.782 & 112.71 & 113.70 & -0.990 \\
\hline \multicolumn{7}{|c|}{ 2-Ethyl-1-hexanol (1) + 2,6-dimethylcyclohexanone (2) } \\
\hline 303.15 & 157.32 & 157.80 & -0.482 & 138.20 & 138.85 & -0.645 \\
\hline 308.15 & 157.86 & 158.51 & -0.653 & 138.69 & 139.51 & -0.822 \\
\hline 313.15 & 158.38 & 159.23 & -0.850 & 139.10 & 140.16 & -1.064 \\
\hline
\end{tabular}

2-ethyl-1-hexanol. Hence, above order was justified. In the present systems, negative values of excess isentropic compressibilities shows that the interaction between the unlike molecules exceed the structure breaking effect between the like molecules.

The negative values of $\kappa_{s}^{E}$ for the four systems are in the following order:

2-Ethyl-1-hexanol + 3-methoxyaniline $>2$-ethyl1-hexanol + 3-methylaniline $>2$-ethyl-1-hexanol + benzyl chloride $>2$-ethyl-1-hexanol +2 , 6-dimethylcyclohexanone

Specific interactions are strong (more effect that is inductive) between 2-ethyl-1-hexanol and 3-methoxyaniline molecules thereby showing the highest negative excess isentropic compressibilities value for their binary mixture.

The sign and magnitude of $\Delta \eta$ and $G^{*}{ }^{*}$ depend on the combined effect of factors such as molecular size, shape, and intermolecular forces [20]. The positive values of $\Delta \eta$ and $G^{*}{ }^{*}$ indicates the presence of specific interactions such as the formation of hydrogen bond between unlike molecules and negative values indicates that mutual loss of specific interactions in like molecules outweigh the specific interactions between unlike molecules $[18,21]$.

In the present study, the positive values of $\Delta \eta$ and $\mathrm{G}^{*} \mathrm{E}$ for these systems indicates that the strength of interaction between the components in binary mixtures and the formation of an association complex [22, 23]. This results in a liquid structure where the flow is rather difficult than would be expected on the basis of the viscosities of the pure components.

\subsection{Partial molar properties}

The interpretations of excess partial molar properties $\left(\bar{V}_{\mathrm{m}, 1^{\prime}}^{\mathrm{E}}\right.$ $\bar{V}_{\mathrm{m}, 2^{\prime}}^{\mathrm{E}}, \bar{K}_{\mathrm{s}, \mathrm{m}, 1}^{\mathrm{E}}$ and $\left.\bar{K}_{\mathrm{s}, \mathrm{m}, 2}^{\mathrm{E}}\right)$ and excess partial molar properties at infinite dilution $\left(\bar{V}_{\mathrm{m}, 1^{\circ}}^{\mathrm{E}}, \bar{V}_{\mathrm{m}, 2^{\prime}}{ }^{\mathrm{E}} \bar{K}_{\mathrm{s}, \mathrm{m}, 1} \mathrm{E}\right.$ and $\left.\bar{K}_{\mathrm{s}, \mathrm{m}, 2} \mathrm{E}\right)$ of components 2 have previously been described [24]. Tables 5 and 6 shows that the values of $\bar{V}_{\mathrm{m}, 1^{\prime}}{ }^{\circ} \bar{V}_{\mathrm{m}, 2^{\prime}}{ }^{\circ} \bar{K}_{\mathrm{s}, \mathrm{m}, 1} \mathrm{E}$ and $\bar{K}_{\mathrm{s}, \mathrm{m}, 2} \mathrm{E}$ are negative over the whole composition range at experimental temperatures. The observed negative values suggest that the hetero molecular association interactions are stronger than the self-association of molecular interactions of like molecules in the mixtures $[25,26]$.

\section{Conclusions}

Densities, viscosities and speeds of sound of binary mixtures of 2-ethyl-1-hexanol with benzyl chloride,3methylaniline,3-methoxyaniline and 2,6-dimethylcyclohexanone have been measured at different temperatures and derived parameters along with their excess/deviations values, and also excess partial molar 
Table 6 The values of $\bar{K}_{\mathrm{s}, \mathrm{m}, 1^{\prime}}^{\circ}$ $K_{\mathrm{s}, \mathrm{m}, 1^{\prime}}^{*} \bar{K}_{\mathrm{s}, \mathrm{m}, 1^{\circ}}{ }^{\mathrm{E}} \bar{K}_{\mathrm{s}, \mathrm{m}, 2^{\prime}}^{\circ} K_{\mathrm{s}, \mathrm{m}, 2}^{*}$ and $\bar{K}_{\mathrm{s}, \mathrm{m}, 2}^{\circ} \mathrm{O}$ of the components 2-ethyl-1-hexanol with various functional groups of binary mixtures at $\mathrm{T}=(303.15-$ 313.15) K

\begin{tabular}{|c|c|c|c|c|c|c|}
\hline $\begin{array}{l}\mathrm{T}(\mathrm{K}) \\
\mathrm{TPa}^{-1}\end{array}$ & $\bar{K}_{\mathrm{s}, \mathrm{m}, 1}^{0}$ & $K_{\mathrm{s}, \mathrm{m}, 1}^{*}$ & $\bar{K}_{\mathrm{s}, \mathrm{m}, 1}^{\circ} \mathrm{E}$ & $\bar{K}_{\mathrm{s}, \mathrm{m}, 2}^{0}$ & $K_{\mathrm{s}, \mathrm{m}, 2}^{*}$ & $\bar{K}_{\mathrm{s}, \mathrm{m}, 2}^{\circ} \mathrm{E}$ \\
\hline \multicolumn{7}{|c|}{ 2-Ethyl-1-hexanol(1) + benzyl chloride (2) } \\
\hline 303.15 & -84.62 & 11.279 & -95.90 & -58.93 & 5.734 & -64.66 \\
\hline 308.15 & -105.33 & 11.739 & -117.07 & -102.61 & 9.753 & -112.36 \\
\hline 313.15 & -130.64 & 12.243 & -142.88 & -175.15 & 19.76 & -194.92 \\
\hline \multicolumn{7}{|c|}{ 2-Ethyl-1-hexanol(1) + 3-methylaniline (2) } \\
\hline 303.15 & -112.46 & 11.279 & -123.74 & -120.22 & 4.534 & -124.75 \\
\hline 308.15 & -127.43 & 11.739 & -139.17 & -136.77 & 4.800 & -141.57 \\
\hline 313.15 & -154.02 & 12.243 & -166.26 & -156.28 & 5.098 & -161.38 \\
\hline \multicolumn{7}{|c|}{ 2-Ethyl-1-hexanol(1) +3-methoxyaniline (2) } \\
\hline 303.15 & -126.03 & 11.279 & -137.31 & -136.70 & 4.151 & -140.85 \\
\hline 308.15 & -143.96 & 11.739 & -155.70 & -153.49 & 4.327 & -157.82 \\
\hline 313.15 & -166.64 & 12.243 & -178.88 & -170.49 & 4.523 & -175.01 \\
\hline \multicolumn{7}{|c|}{ 2-Ethyl-1-hexanol(1) +2,6-dimethylcyclohexanone(2) } \\
\hline 303.15 & -16.76 & 11.279 & -28.04 & -11.11 & 8.965 & -20.08 \\
\hline 308.15 & -20.09 & 11.739 & -31.83 & -14.04 & 9.338 & -23.38 \\
\hline 313.15 & -23.69 & 12.243 & -35.94 & -17.51 & 9.725 & -27.23 \\
\hline
\end{tabular}

properties at infinite dilution $\left(\bar{V}_{\mathrm{m}, 1^{\circ}}^{\circ}, \bar{V}_{\mathrm{m}, 2}^{\circ}{ }^{\circ}, \bar{K}_{\mathrm{s}, \mathrm{m}, 1}^{\circ \mathrm{E}}\right.$ and $\left.\bar{K}_{\mathrm{s}, \mathrm{m}, 2}^{\circ}\right)$ were calculated. The results are analyzed in terms of the specific interactions through the hetero molecular association between the components of the mixtures, resulting in the formation of association complexes.

\section{Compliance with ethical standards}

Conflict of interest The authors declare that they have no conflict of interest.

\section{References}

1. Prasad N, Prakash S (1976) Solute-water interactions and the solubility behaviour of long-chain paraffin hydrocarbons. Acustica 36:313-319

2. Deshpande DD, Bhatgadde LG (1968) Sound velocities, adiabatic compressibilities and free volumes in aniline solutions. J Phys Chem 72(1):261-266

3. Snyder WJ, Snyder JR (1974) Velocity of sound in binary mixtures of benzene, hexane, and methanol at 0-65 Deg. J Chem Eng Data 19(3):270-274

4. Naga Babu P, Venkata Lakshmi V, Gowrisankar M, Raveendra M (2019) Investigation of molecular interactions in binary mixtures of 2-methoxyaniline with isomeric cresols at various temperatures through thermo physical properties. Phys Chem Liq. https ://doi.org/10.1080/00319104.2019.1594225

5. Sulthana SP, Gowrisankar M, Babu S, Santos D (2019) Investigation of ketonic effect in molecular interactions of 2-methylcyclohexanone with aniline, $\mathrm{N}$-methyl aniline and $\mathrm{N}, \mathrm{N}$-dimethyl aniline at various temperatures. Int J Ambient Energy. https:// doi.org/10.1080/01430750.2019.1673816
6. Mukesh B, Sreekanth T, Srinivasa Krishna T, Gowrisankar M (2019) Thermodynamic and transport properties of 2-methoxyaniline with substituted ethanols at various temperatures. Int J Ambient Energy. https://doi.org/10.1080/01430750.2019.1636873

7. Bhatia SC, Sangwa J, Rani R, Kiran V (2013) Densities, viscosities, speeds of sound, and refractive indices of binary mixtures of 2-ethyl-1-hexanol with benzene and halobenzenes. Int J Thermophys 34(11):2076-2088

8. Zorȩbski E, Dzida M, Wysocka E (2011) Acoustic and thermodynamic properties of 2-ethyl-1-hexanol by means of highpressure speed of sound measurements at temperatures from (293 to 318) K and pressures up to $101 \mathrm{MPa}$. J Chem Eng Data 56(5):2680-2686

9. Zábranský M, Růžička V Jr (2004) Estimation of the heat capacities of organic liquids as a function of temperature using group additivity: an amendment. J Phys Chem 33:1071-1081

10. Pandiyan V, Oswal SL, Vasantharani P (2011) Thermodynamic and acoustic properties of binary mixtures of ethers. III. Diisopropyl ether or oxolane with o- or m-toluidines at 303.15, 313.15 and 323.15 K. Thermochim Acta 516:64-73

11. Reid RC, Prausnitz JM, Poling BE (1987) The properties of gases and liquids. McGraw Hill Inc., New York, p 139

12. Riddick JA, Bunger WB, Sakano TK (1986) Organic solvents physical properties and methods of purification, 4th edn. Wiley Interscience, New York

13. Kalavathi E, Venkatesulu A, Gowrisankar M (2019) Influence of carbonyl group on thermodynamic and transport properties of binary liquid mixtures of 2-methoxyaniline with ketones at various temperatures. Chem Data Collect 23:100260

14. Ali A, Nain AK, Chand D, Lal B (2005) Molecular interactions in binary mixtures of anisole with benzyl chloride, chlorobenzene and nitrobenzene at $303.15 \mathrm{~K}$ : an ultrasonic, volumetric, viscometric and refractive index study. Ind J Chem 44A:511-515

15. Venkateswara Rao P, Gowrisankar M, Venkatramana L, Srinivasa Krishna T, Ravindhranath K (2016) Studies on the importance of nature of substituent on the thermodynamic and transport properties of liquid mixtures at various temperatures. J Chem Therm 101:92-102 
16. Benson GC, Kiyohara O (1979) Evaluation of excess isentropic compressibilities and isochoric heat capacities. J Chem Therm 11:1061-1064

17. Redlich O, Kister AT (1948) Algebraic representation of thermodynamic properties and the classification of solutions. J Ind Eng Chem 40:345-348

18. Fort RJ, Moore WR (1966) Viscosities of binary liquid mixtures. Trans Faraday Soc 62:1112-1119

19. Nigam RK, Singh PP, Singh M, Singh KC (1980) Excess enthalpies of mixing of aniline + isomeric xylenes \& Barker's theory of associated mixtures. Indian J Chem 19A(3):192-194

20. Nath J, Srivastava AK (1986) Excess volumes for binary liquid mixtures of trichloroethylene with anisole, pyridine, quinoline and cyclohexane at 298.15 and 308.15 K. Fluid Phase Equili 28(1):97-101

21. Iloukhani H, Reddy KD, Prabhakara Rao MV (1985) Excess volumes of trichloroethylene with some aliphatic and alicyclic ketones at 303.15 K and 313.15 K. Phys Chem Liq 14(3):181-188

22. Reed TM III, Taylor TE (1959) Viscosities of liquid mixtures. J Phys Chem 63(1):58-67

23. Meyer R, Meyer M, Metzer J, Peneloux A (1971) Thermodynamic and physicochemical properties of binary solvent. J Chem Phys 62:405

24. Gowrisankar M, Venkateswarlu P, Kumar KS, Sivarambabu $S$ (2012) Thermodynamics of amine + ketone mixtures 3.
Volumetric, speed of sound data and viscosity at (303.15 and $308.15 \mathrm{~K}$ ) for the binary mixtures of $\mathrm{N}, \mathrm{N}$-dimethylaniline + propiophenone, $+\mathrm{p}$-methylacetophenone, $+\mathrm{p}$-chloroacetophenone. Mol Liq 173:172-179

25. Fernandez J, Andrade MP, Pintos M, Sarmiento F, Bravo R (1983) Excess enthalpies of (secondary amine + alcohol) at $298.15 \mathrm{~K} . \mathrm{J}$ Chem Therm 15(6):581-584

26. Rao DN, Naidu PR (1981) Excess volumes of (ethylenediamine + an alcohol). J Chem Therm 13(7):691-694

27. Busygina GI, Maslova VA, Shvetsova KG, Babinkov AG, Rabinovich IB, Karyakin NV (1987) Specific heat and thermodynamic functions of phthalic anhydride and 2-ethylhexanol at 13-350 K. Zhur Fiz Khim 61:2347-2351

28. Smith RH, Andrews DH (1931) Thermal energy studies. I. Phenyl derivatives of methane, ethane and some related compounds. J Am Chem Soc 53:3644-3660

Publisher's Note Springer Nature remains neutral with regard to jurisdictional claims in published maps and institutional affiliations. 